\title{
Perfil de mulheres com incontinência urinária submetidas a procedimento cirúrgico em um hospital de ensino do sul do país
}

\section{Profile of women with urinary incontinence who underwent surgical procedure in a teaching hospital in the southern Country}

\author{
Juliana Cristina Frare ${ }^{1}$; Francieli Tibes de Souza ${ }^{2}$; Joseane Rodrigues da Silva ${ }^{3}$
}

\section{Resumo}

\begin{abstract}
A incontinência urinária (IU) é definida como qualquer perda involuntária de urina pela uretra, possível de ser caracterizada como um problema social e/ou higiênico. O tratamento da IU pode ser realizado cirurgicamente ou por meio de técnicas conservadoras como a fisioterapia, a terapia comportamental e medicamentosa. O objetivo da pesquisa foi identificar o perfil sociodemográfico e clínico de mulheres que realizaram procedimento cirúrgico para correção de IU no Hospital Universitário do Oeste do Paraná (HUOP). Foram analisados todos os prontuários das pacientes atendidas no setor de ginecologia com diagnóstico de IU submetidas a tratamento cirúrgico nos anos de 2008 e 2009. Os dados foram lançados em uma planilha do programa Excel 7.0 (Microsoft ${ }^{\circledR}$ ), e posteriormente analisados em termos de freqüências relativas e absolutas. Os resultados demonstraram que a idade média das pacientes foi de 52,41 anos $( \pm 12,11)$, dos quais $128(58,99 \%)$ tinham entre 40 e 59 anos. A incontinência urinária de esforço (IUE) foi o tipo de IU mais prevalente, tendo sido verificado em $193(88,94 \%)$ dos 217 prontuários analisados. A técnica cirúrgica mais utilizada foi a do sling pubovaginal associada à colpoperineoplastia (CPP) anterior, realizada em $117(53,92 \%)$ pacientes. Somente seis $(2,76 \%)$ prontuários relataram a atuação da fisioterapia nas pacientes com IU submetidas a tratamento cirúrgico.

Palavras-Chave: Incontinência urinária. Cirurgia. Fisioterapia.
\end{abstract}

\begin{abstract}
Urinary incontinence (UI) is defined as any involuntary loss of urine that may characterize a social and/ or hygienic problem. The treatment of UI can be performed surgically or by conservative techniques such as physiotherapy, behavioral therapy and drug therapy. The research aim was to identify the sociodemographic and clinical profile of women who performed surgery procedure for correction of UI at the Western University Hospital of Paraná (HUOP). All records of patients treated at the gynecology sector diagnosed with UI undergoing surgery in the years 2008 and 2009 were analyzed. Data was entered into a spreadsheet and analyzed in terms of relative and absolute frequencies. The results demonstrate that the average was observed in 52.41 years $( \pm 12.11), 128(58.99 \%)$ patients were aged between 40 and 59 years old. The stress urinary incontinence (UI) was the most prevalent disease, observed in 193 (88.94\%) of 217 records analyzed. The surgical techniques used in most cases was pubovaginal sling associated of colpoperineoplastia (CPP), realized in 117 (53.92\%) patients. Only six (2.76\%) records reported the physiotherapy performance in women with UI submitted to surgery treatment.
\end{abstract}

Key words: Urinary incontinence. Surgery. Physiotherapy.

1 Universidade Estadual do Oeste do Paraná/Docente, Brasil. Fisioterapeuta, Docente da disciplina de Fisioterapia em Saúde da Mulher, Colegiado de Fisioterapia, Centro de Ciências Biológicas e da Saúde Contato principal para correspondência. jcfrare@ yahoo.com.br.

2 Universidade Estadual do Oeste do Paraná/Graduada. Fisioterapeuta, Graduada pela Universidade Estadual do Oeste do Paraná. fran_ftds@hotmail.com.

3 Universidade Estadual do Oeste do Paraná/Docente, Brasil. Fisioterapeuta, Docente da disciplina de Fisioterapia em Geriatria e Gerontologia.joseane_rs@yahoo.com.br 


\section{Introdução}

A incontinência urinária (IU) é definida como qualquer perda involuntária de urina pela uretra, possível de ser caracterizada como um problema social e/ou higiênico (ABRAMS et al., 2003). É uma patologia de importância pela sua alta incidência, visto que, acomete cerca de 30 a $60 \%$ de todas as mulheres durante o período de climatério e na menopausa, afetando negativamente suas vidas (GUARISI et al., 2001a).

Essa patologia é classificada em três tipos, a incontinência urinária de esforço (IUE), causada pela deficiência esfincteriana ou pela hipermobilidade da uretra, a incontinência urinária de urgência (IUU), secundária à instabilidade do músculo detrusor, e a incontinência urinária mista (IUM) associada à urgência e às situações de aumento da pressão intraabdominal, ou seja, uma combinação entre os dois tipos descritos anteriormente (FELDNER JUNIOR et al., 2002).

A IUE frequentemente está associada ao prolapso genital, definido como deslocamento das vísceras pélvicas no sentido caudal em direção ao hiato genital, sendo classificado em: prolapso uterino, prolapso de parede vaginal anterior (cistocele ou uretrocele) e prolapso de parede vaginal posterior (retocele ou enterocele). O deslocamento das vísceras pélvicas pode ser total ou parcial, e decorrente do desequilíbrio entre as forças que mantêm os órgãos pélvicos em sua posição normal e aquelas que tendem a empurrá-los para fora (GONÇALVES et al., 1997).

Os episódios de IU, assim como o prolapso genital, são causadores de constrangimento social, disfunção sexual e baixo desempenho profissional. No entanto, muitas mulheres consideram esta patologia uma condição normal e resultado do processo de envelhecimento, fato que as leva a não procura por tratamento e, quando o fazem, em geral, é tardiamente (HIGA; LOPES; REIS, 2008; LOPES; HIGA, 2006).
O tratamento da IU pode ser realizado cirurgicamente ou por meio de técnicas conservadoras como a fisioterapia, a terapia comportamental e medicamentosa (SILVA; LOPES, 2009).

Várias técnicas cirúrgicas têm sido empregadas para correção da IU, destacando-se a técnica do sling (suporte) pubovaginal, que é colocado na região suburetral e tem como finalidade restaurar a normalidade anatômica e funcional da bexiga dando suporte e estabilizando a uretra sem obstruí-la. Para isso, é utilizada uma faixa de material autólogo ou heterólogo, contra a qual a uretra é forçada durante o aumento da pressão abdominal, fechando-se e mantendo a continência (HAAB; LEACH, 1999; SARTORI et al., 2008).

Quanto ao tratamento cirúrgico dos prolapsos vaginais, já foram descritas várias técnicas diferentes, podendo as mesmas ser realizadas via vaginal, abdominal ou laparoscópica (ZUCHI et al., 2003). A colpoperineoplastia (CPP) é um procedimento cirúrgico realizado via vaginal que consiste em elevar a uretra e o colo vesical, por meio da aproximação das bordas do músculo diafragma urogenital na linha média, restaurando o assoalho pélvico lesado. Dessa forma, não há o deslocamento excessivo da fáscia endopélvica sob a uretra, o que permite o fechamento da mesma durante o aumento da pressão intra-abdominal (WALL, 1998).

Apesar de existirem inúmeras técnicas cirúrgicas para correção da IU, estas não são efetivas em todos os casos, além do que pode ocorrer recidiva dos sintomas em um tempo inferior a 5 anos (BERNARDES et al., 2000). São descritos diversos fatores associados a recidivas de IUE após correção cirúrgica, entre eles: cirurgia pélvica prévia, realização de perineoplastia posterior associada, idade, história obstétrica, obesidade, constipação, tosse crônica, hipoestrogenismo, habilidade e familiaridade do cirurgião com a técnica cirúrgica utilizada (RAMOS et al., 2000).

Nos casos em que a paciente já foi previamente 
submetida à cirurgia ginecológica, as chances de ter novamente o problema são bem maiores, como encontrado no estudo realizado por Ramos et al. (2000), no qual houve recidiva em 100\% das pacientes com cirurgia prévia, dois anos após o procedimento.

Devido aos altos índices de insucesso nos tratamentos cirúrgicos, nos últimos anos a fisioterapia vem ganhando maior projeção, sendo considerada uma técnica de baixo custo, baixo risco e poucos efeitos colaterais, além da eficácia comprovada. Em 2005, a Sociedade Internacional de Continência indicou a fisioterapia como opção de primeira linha para IU, contudo, no Brasil, poucos serviços públicos dispõem deste tipo de atendimento direcionado às mulheres incontinentes. Mencionamse como objetivos no tratamento fisioterapêutico a reeducação dos músculos do assoalho pélvico e seu fortalecimento, por meio de recursos como a cinesioterapia, eletroestimulação, biofeedback, cones vaginais e ginástica hipopressiva (ABRAMS et al., 2005; FIGUEIREDO et al., 2008; TEIXEIRA et al., 2005).

Conhecer o perfil das mulheres incontinentes permite aos profissionais da saúde obter informações da real dimensão do problema e sua repercussão na população. Dessa forma, é possível auxiliar o desenvolvimento de ações preventivas e reabilitadoras, possibilitando uma assistência fisioterapêutica uroginecológica mais eficiente e segura.

Assim, este estudo teve como objetivo identificar o perfil sociodemográfico e clínico de mulheres que realizaram procedimento cirúrgico para correção de IU no Hospital Universitário do Oeste do Paraná nos anos de 2008 e 2009.

\section{Material e Métodos}

Trata-se de um estudo transversal, retrospectivo, de caráter quantitativo. A coleta de dados foi realizada no Serviço de Apoio Médico Estatístico
(SAME) do Hospital Universitário do Oeste do Paraná (HUOP) na cidade de Cascavel - PR, no período de abril a julho de 2010. Foram analisados todos os prontuários das pacientes atendidas no setor de ginecologia do HUOP com diagnóstico de IU submetidas a tratamento cirúrgico no período de janeiro de 2008 a dezembro de 2009.

Foram coletadas informações relativas à identificação das pacientes, diagnóstico, tempo de perda urinária, história obstétrica, procedimentos realizados, cirurgias ginecológicas prévias, patologias associadas e abordagem fisioterapêutica, utilizando-se uma ficha previamente elaborada. Todos os dados foram lançados em uma planilha do programa Excel 7.0 (Microsoft $®)$, e posteriormente analisados em termos de freqüências relativas e absolutas.

Este trabalho recebeu aprovação do Comitê de Ética em Pesquisa da Universidade Estadual do Oeste do Paraná, Unioeste - PR, em atendimento à Resolução 196/96 do Conselho Nacional de Saúde. Parecer n 065/2010 - CEP.

\section{Resultados e Discussão}

O HUOP atende predominantemente pacientes usuárias do Sistema Único de Saúde (SUS). No período de pesquisa foram analisados 217 prontuários arquivados no SAME de pacientes que foram atendidas no setor de ginecologia do HUOP para tratamento cirúrgico da IU nos anos de 2008 e 2009. Em função das informações incompletas nos prontuários, dados referentes ao tempo de perda urinária e história obstétrica foram excluídos.

A idade média das pacientes observada nos prontuários foi de 52,41 anos $( \pm 12,11)$, sendo que $61(28,11 \%)$ pacientes tinham idade entre 40 e 49 anos e 67 (30,88\%) entre 50 e 59 anos. A idade mínima encontrada foi de 20 anos e a máxima de 88 anos (Tabela 1). 
Tabela 1 - Distribuição das pacientes de acordo com a idade, Cascavel, 2010.

\begin{tabular}{lcc}
\hline Idade (anos) & F & $\mathbf{f} \%$ \\
\hline $20-29$ & 3 & 1,38 \\
$30-39$ & 29 & 13,36 \\
$40-49$ & 61 & 28,11 \\
$50-59$ & 67 & 30,88 \\
$60-69$ & 39 & 17,98 \\
$70-79$ & 15 & 6,91 \\
$80-89$ & 3 & 1,38 \\
\hline Total & 217 & 100 \\
\hline
\end{tabular}

Este resultado é similar ao descrito por Figueiredo et al. (2008), que investigaram 63 prontuários de mulheres com IU atendidas no serviço de Fisioterapia Uroginecológica do Hospital das Clínicas da Universidade Federal de Minas Gerais, e encontraram maior prevalência de IU na faixa etária de 40 e 59 anos (81\%).

Segundo Lopes e Higa (2006), mulheres mais jovens, em torno dos 45 anos, geralmente apresentam queixa de perda urinária aos esforços, enquanto que, mulheres com idade mais avançada queixam-se de urgência miccional, a qual pode ou não estar associada à perda aos esforços.

Neste estudo, $128 \quad(58,99 \%)$ pacientes que realizaram cirurgia para correção da IU, encontravam-se na faixa etária entre 40 e 59 anos, havendo, portanto, predomínio de mulheres de meia-idade atendidas no HUOP.

Embora a IU não seja resultado do processo de envelhecimento, a idade avançada tem sido apontada como uma das causas desta patologia. As mulheres idosas têm maiores riscos de desenvolver o problema, já que, com o tempo, ocorre uma diminuição na força muscular do períneo, em conseqüência do decréscimo na espessura dessa musculatura. Além disso, a capacidade da bexiga diminui, passando de 500 a $600 \mathrm{ml}$ para 250 a 300 $\mathrm{ml}$, e há uma diminuição dos níveis de estrogênio em decorrência da menopausa, o que predispõe a IU na mulher idosa (MATHEUS et al., 2006; HIGA; LOPES; REIS, 2008).

Do total de pacientes deste estudo, somente 57 (26,27\%), tinham idade superior a 60 anos, evidenciando que, a procura por este tipo de atendimento no HUOP é relativamente menor entre as mulheres idosas.

Segundo alguns autores, uma das razões para não procura por tratamento é o fato de a IU ser considerada como parte integrante do processo de envelhecimento e, por isso, acredita-se que a perda de urina é algo normal, não merecendo atenção dos profissionais da saúde (SILVA; LOPES; 2009; GUARISI et al., 2001a; LOPES; HIGA, 2006).

Quanto à raça, observou-se neste estudo que 195 $(89,86 \%)$ pacientes eram de cor branca, $19(8,76 \%)$ negras e três $(1,38 \%)$ pardas (Tabela 2$)$. O mesmo foi encontrado no artigo de Beltrami et al. (2009), os quais avaliaram o atendimento de pacientes com IU em um serviço universitário e verificaram que $84,6 \%$, em um total de 26 , eram de cor branca. Também Retti et al. (2007), em seu estudo com 26 mulheres, observaram que $76,9 \%$ das avaliadas eram brancas.

Acredita-se que existam determinantes genéticos que protegem as mulheres negras da IU. Alguns autores apontam que, as diferenças na anatomia ou na resistência da uretra e nas estruturas de suporte do assoalho pélvico, sejam os responsáveis pela menor taxa de incidência da IU nas mulheres negras (BROWN et al., 1999; HIGA; LOPES; REIS, 2008; GRODSTEIN et al., 2003). Em contrapartida, um estudo realizado por Guarisi et al. (2001b) observou que na população brasileira, devido a miscigenação racial, as distinções raciais com base na cor da pele não são precisas, dificultando o estabelecimento desta diferença.

Em relação ao estado civil, 125 (57,61\%) eram casadas, $25(11,52 \%)$ viúvas, 20 (9,22\%) solteiras, $17(7,83 \%)$ divorciadas, oito $(3,68)$ união consensual, seis separadas $(2,77 \%), 16(7,37 \%)$ 
não informado. Quando pesquisado sobre a religião, $129(59,45 \%)$ pacientes eram católicas, $44(20,28 \%)$ evangélicas, $28(12,90 \%)$ de outras religiões e 16 $(7,37 \%)$ não foi informado (Tabela 2).

O predomínio de mulheres, casadas e da religião católica com IU também foi observado no estudo de Beltrami et al. (2009), no qual aproximadamente 13 , das 26 pacientes convivia com parceiro e possuíam religião católica.

Com relação à profissão, 100 (46,08\%) pacientes eram do lar, 14 (6,45\%) costureiras, 13 $(5,99 \%)$ aposentadas, sete $(3,23 \%)$ agricultoras, seis $(2,76 \%)$ zeladoras, quatro $(1,85 \%)$ domésticas, 39 (17,97\%) tinham outras profissões e 34 (15,67\%) não informado (Tabela 2).

Quanto à cidade de origem, 177 (81,57\%) pacientes eram da cidade de Cascavel e 40 $(18,43 \%)$ residiam em cidades da região. Também se verificou que $201(92,63 \%)$ prontuários eram de pacientes residentes na zona urbana e $16(7,37$ \%) na zona rural (Tabela 2).

Acredita-se que o predomínio de pacientes procedentes da zona urbana atendidas pelo serviço seja devido à maior facilidade de acesso das pacientes aos meios de informação e aos serviços públicos de saúde disponíveis, quando comparado às que residem na zona rural.

O HUOP caracteriza-se como sendo um hospital público e também de ensino. Promove atendimento especializado em diversas áreas da Medicina, dentre elas a ginecologia, servindo de referência na atenção em saúde, para quatro regionais da Secretaria de Saúde do Estado do Paraná. As pacientes são inicialmente atendidas nas Unidades Básicas de Saúde (UBS) das cidades de origem, tanto na zona urbana quanto na zona rural, e posteriormente encaminhadas para o ambulatório de ginecologia do HUOP, onde os tratamentos são definidos. Isso justifica a diversidade nas procedências de pacientes verificada nesse estudo, bem como o grande número de procedimentos cirúrgicos realizados no período do estudo.

O diagnóstico de IUE foi verificado em 193 $(88,94 \%)$ pacientes, seguido por IUM em 22 $(10,14 \%)$ e IUU em dois $(0,92 \%)$ pacientes.

Souza et al. (2009), em estudo que comparou a função muscular do assoalho pélvico em 153 mulheres continentes e incontinentes, com idades entre 78 e 87 anos, observaram que $41,7 \%$ da sua amostra apresentavam IUE, 34,5\% com IUM e 23,8\% com IUU.

Em contrapartida, estudo realizado por Figueiredo et al. (2008), no qual se caracterizou o perfil clínico e sociodemográfico de 58 mulheres com IU atendidas em um serviço público de fisioterapia uroginecológica, observou-se que a IUM prevaleceu em $63 \%$ da amostra.

Também Beltrami et al. (2009), que analisaram 26 pacientes com queixa de perda urinária encaminhadas de unidades básicas de saúde, verificaram que mais da metade $(69,2 \%)$ apresentava IUM.

Dedicação et al. (2009), em um estudo retrospectivo, compararam o impacto do tipo de IU sobre a qualidade de vida em 77 mulheres que realizaram tratamento fisioterapêutico para IU. No estudo, o exame urodinâmico foi utilizado para classificar a IU, e observou-se que a maioria das mulheres $(44,16 \%)$ apresentou IUM, 40,26\% foram diagnosticadas como IUE e $15,58 \%$ como IUU.

O diagnóstico de cistocele grau II foi encontrado em $72 \quad(33,18 \%)$ prontuários, cistocele grau I em 51 (23,50\%), retocele grau II em 33 (15,21\%), retocele grau I em 27 (12,44\%), cistocele grau III em 18 (8,29\%), mioma uterino em $15(6 \%)$, retocele grau III em nove $(4,15 \%)$, prolapso uterino em nove $(4,15 \%)$. Delas, sete $(3,23 \%)$ tinham prolapso uterino grau I. A rotura perineal foi observada em cinco $(2 \%)$ pacientes (Figura 1). 
Tabela 2 - Distribuição das pacientes de acordo com as características sócio-demográficas, Cascavel, 2010.

\begin{tabular}{|c|c|c|}
\hline Características & $\mathbf{F}$ & $f \%$ \\
\hline \multicolumn{3}{|l|}{ Raça } \\
\hline Branca & $\underline{195}$ & $\underline{89,86}$ \\
\hline Negra & 19 & 8,76 \\
\hline Parda & 3 & 1,38 \\
\hline \multicolumn{3}{|l|}{ Estado civil } \\
\hline Casadas & $\underline{125}$ & $\underline{57,61}$ \\
\hline Viúvas & 25 & 11,52 \\
\hline Solteiras & 20 & 9,22 \\
\hline Divorciadas & 17 & 7,83 \\
\hline União consensual & 8 & 3,68 \\
\hline Separadas & 6 & 2,77 \\
\hline Não informado & 16 & 7,37 \\
\hline \multicolumn{3}{|l|}{ Religião } \\
\hline Católica & $\underline{129}$ & $\underline{59,45}$ \\
\hline Evangélica & 44 & 20,28 \\
\hline Outras & 28 & 12,90 \\
\hline Não informado & 16 & 7,37 \\
\hline \multicolumn{3}{|l|}{ Ocupação } \\
\hline Do lar & $\underline{100}$ & $\underline{46,08}$ \\
\hline Costureira & 14 & 6,45 \\
\hline Aposentada & 13 & 5,99 \\
\hline Agricultora & 7 & 3,23 \\
\hline Zeladora & 6 & 2,76 \\
\hline Empregada Doméstica & 4 & 1,85 \\
\hline Outras & 39 & 17,97 \\
\hline Não informado & 34 & 15,67 \\
\hline \multicolumn{3}{|l|}{ Cidade de Origem } \\
\hline Cascavel & $\underline{177}$ & $\underline{81,57}$ \\
\hline Vera Cruz do Oeste & 8 & 3,69 \\
\hline Quedas do Iguaçu & 5 & 2,30 \\
\hline Nova Aurora & 4 & 1,84 \\
\hline Céu Azul & 4 & 1,84 \\
\hline Corbélia & 4 & 1,84 \\
\hline Catanduvas & 3 & 1,38 \\
\hline Outras & 12 & 5,54 \\
\hline \multicolumn{3}{|l|}{ Zona de procedência } \\
\hline Urbana & $\underline{201}$ & $\underline{92,63}$ \\
\hline Rural & 16 & 7,37 \\
\hline Total & 217 & 100 \\
\hline
\end{tabular}

$\mathrm{F}=$ frequência em número; $f \%=$ frequência em porcentagem. 
Figura 1 - Distribuição das pacientes de acordo com o diagnóstico clínico. Cascavel, PR, 2010.

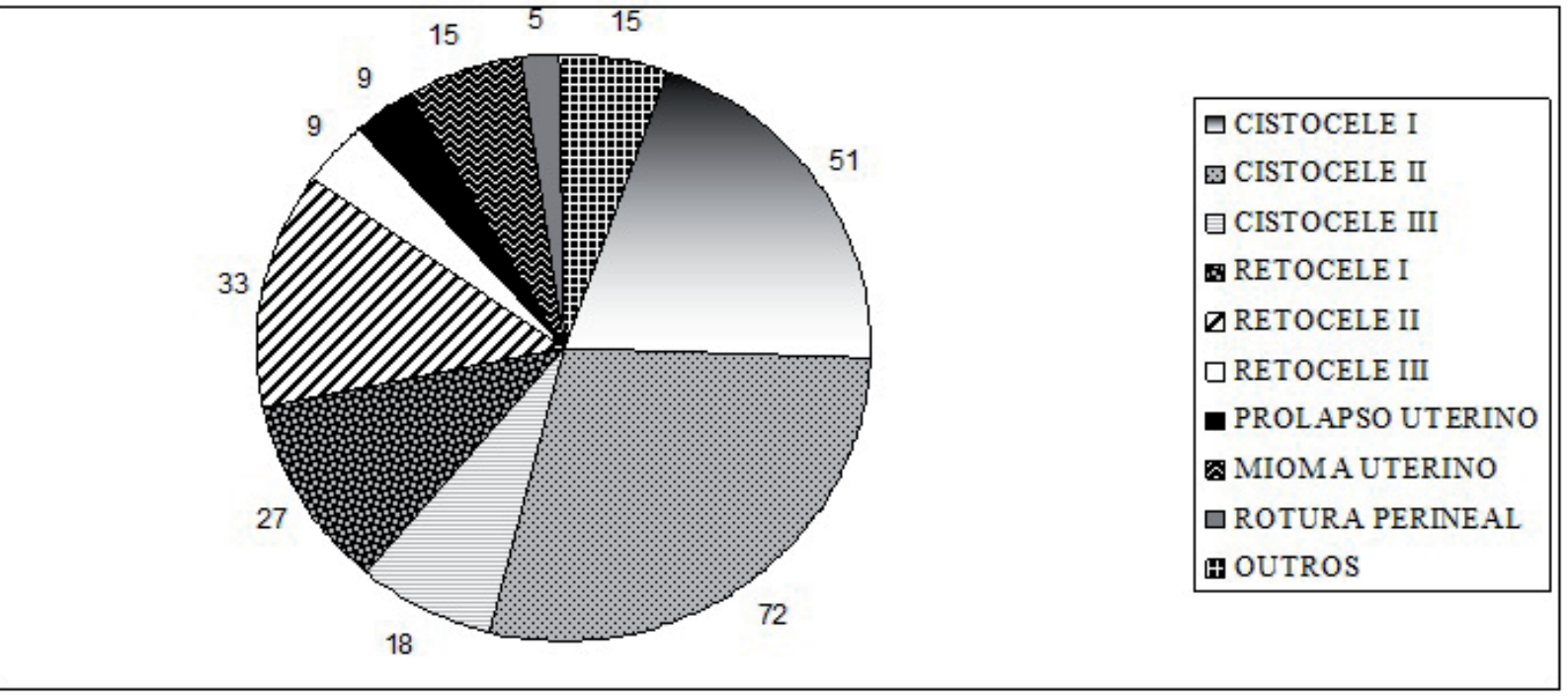

Durante a coleta de dados deste estudo, não havia nos prontuários relato de exames complementares para diagnóstico e classificação da IU. No geral, o mesmo era realizado baseado na história clínica das pacientes e exame físico. Dados referentes ao tempo de perda urinária e história obstétrica, fundamentais para diagnóstico desta patologia, tiveram que ser excluídos do estudo, devido ao grande número de prontuários que não continham essas informações.

Segundo Feldner Junior et al. (2002), a história clínica e o exame físico não devem ser utilizados como único meio diagnóstico da IU, uma vez que existem recursos mais confiáveis para isso, como o estudo urodinâmico. Aliás, um diagnóstico incorreto pode ter como conseqüência a indicação cirúrgica imprópria ou até desnecessária.

Quando pesquisadas as patologias associadas, a hipertensão arterial sistêmica (HAS) estava presente em 68 (31,64\%) pacientes deste estudo, patologias da tireóide em oito (3,69\%), HAS associada à patologia da tiróide em seis $(2,76 \%)$, diabetes mellitus (DM) em uma (0,46\%), DM associada à HAS em cinco (2,30\%), e 129 (59,45\%) pacientes não possuíam patologias associadas. No total, a HAS foi observada em $79(36,41 \%)$ pacientes.
Segundo Moller, Lose e Jorgensen (2000), há hipótese de que a carga excessiva na bexiga, após tratamento para HAS com diuréticos, contribui para a IU na mulher.

Quanto aos procedimentos cirúrgicos realizados para correção da IU, observou-se, neste estudo, que a técnica do sling pubovaginal sintético (SPS), associada à colpoperineoplastia (CPP) anterior, foi a mais utilizada, sendo aplicada em 117 (53,92\%) pacientes. Ela foi seguida pelo SPS associado à CPP anterior e posterior em 60 (27,65\%), SPS em 18 (8,29\%), CPP anterior e posterior em 12 (5,53\%), SPS associado à CPP posterior em seis $(2,77 \%)$, CPP anterior em duas $(0,92 \%)$ e CPP posterior em dois $(0,92 \%)$ (Figura 2).

Segundo Oliveira et al. (2003), a técnica de sling pubovaginal apresenta-se como a melhor opção no tratamento da IUE pelos baixos índices de recidiva e facilidade na execução da cirurgia, sendo poucas as complicações e intercorrências observadas. Os slings sintéticos foram desenvolvidos com o intuito de tratar a IUE de forma menos invasiva e com menor incidência de distúrbios miccionais no pósoperatório (SARTORI et al., 2008).

Em estudo realizado por Silveira et al., (2007), 
composto por 47 pacientes submetidas ao tratamento da IUE com sling sintético de polipropileno, obtevese uma taxa de cura em $92 \%$ das pacientes, mantendo a continência após um período de três meses de acompanhamento. Esses mesmos autores concluem que é necessário um período de acompanhamento maior, entretanto a técnica em questão mostrou-se aparentemente eficaz.

De acordo com Oliveira et al. (2003), a escolha do procedimento cirúrgico deve levar em consideração o correto diagnóstico, os índices de recidivas, a integridade do sistema esfincteriano uretral, assim como a experiência do cirurgião com a técnica.

Figura 2 - Distribuição das pacientes de acordo com os procedimentos cirúrgicos realizados. Cascavel, PR, 2010.

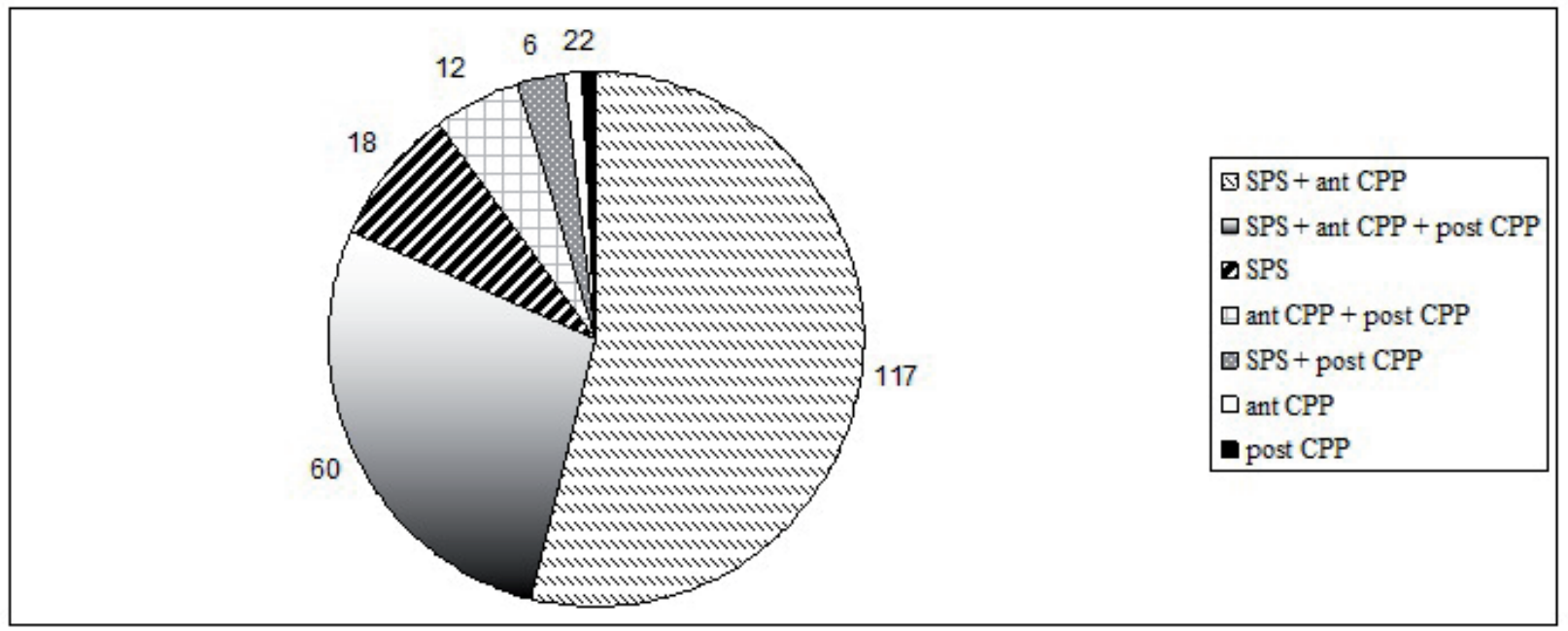

Quanto ao tempo de internação, verificou-se que as pacientes permaneceram internadas em média $2,75( \pm 0,90)$ dias, e $102(47 \%)$ permaneceram internadas por três dias e $82(37,79 \%)$ por dois dias, o período mínimo de internamento foi de um dia e o máximo de seis dias.

Semelhante ao encontrado, Meloni et al. (2004) observaram em estudo retrospectivo com 27 mulheres com diagnóstico de IUE, que o tempo de internação das pacientes submetidas a correção cirúrgica pela técnica sling pubovaginal foi, em média, 2,4 dias.

O procedimento cirúrgico para correção da IU, realizado através dos SPS, implica menor tempo de internação, além de ser uma técnica com menores índices de dor e infecção na ferida pós-operatória. Ainda assim, esse procedimento, apesar de menos invasivo, pode ocasionar complicações e ser contraindicado para algumas mulheres, além dos custos serem elevados (RETTI, et al., 2007). Estima-se que nos Estados Unidos os gastos com IU se aproxime dos 16 milhões de dólares por ano. No Brasil, não há estimativas quanto aos gastos anuais (BELTRAMI et al., 2009; DEDICAÇÃO et al., 2009).

Em relação às cirurgias prévias, do total de prontuários analisados, 82 (37,79\%) eram de pacientes que já tinham realizado um ou mais tipos de cirurgia ginecológica anteriormente. 46 (50,59\%) pacientes já haviam sido submetidas à cirurgia para tratamento de IU, e três $(6,52 \%)$ destas tinham mais de duas cirurgias prévias. A histerectomia havia sido realizada em 29 (25,37\%) pacientes, laqueadura em $22(26,83 \%)$, outros tipos de cirurgias ginecológicas em sete $(8,54 \%)$ (Figura 3$)$. 
Figura 3 - Distribuição das pacientes de acordo com as cirurgias ginecológicas previamente realizadas. Cascavel, PR, 2010.

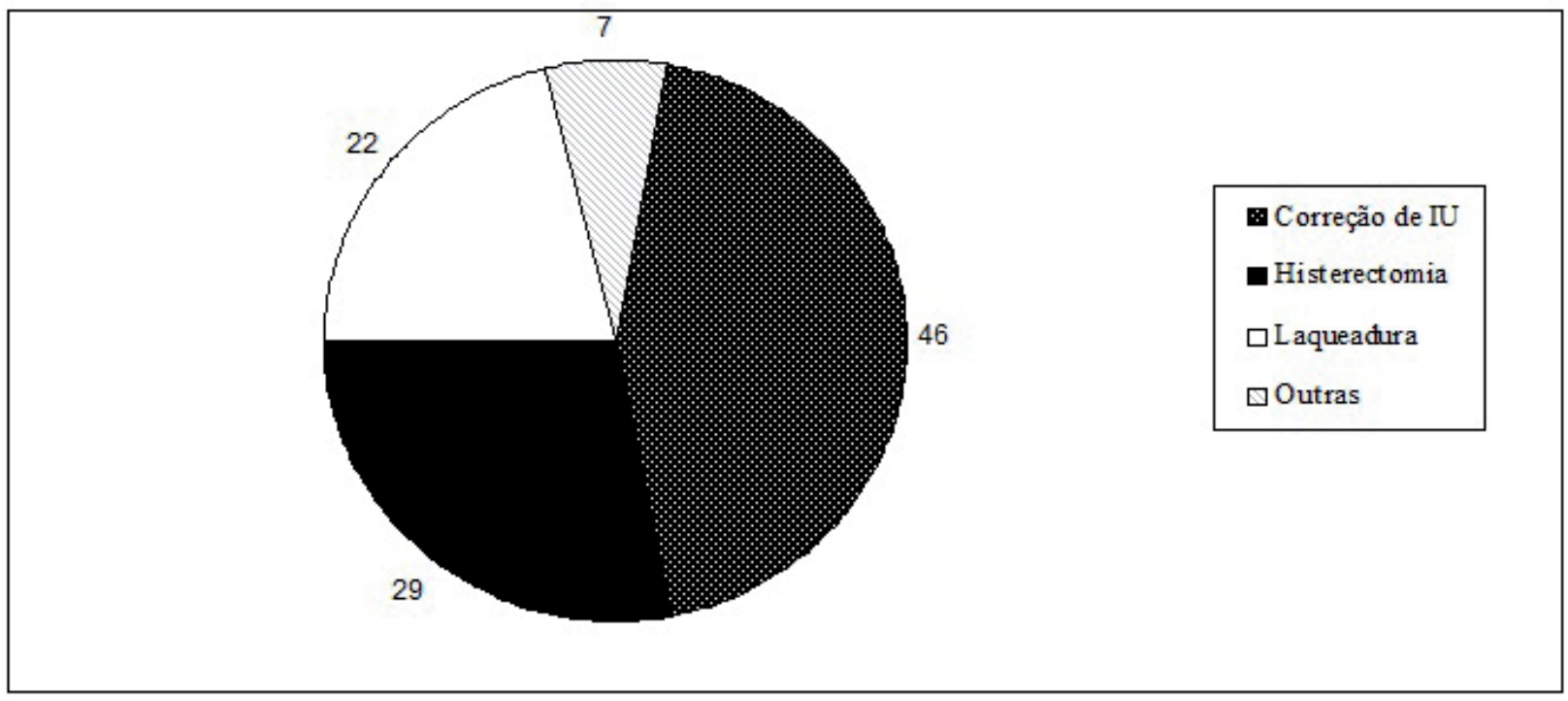

Outros artigos também mencionam os casos de recorrência cirúrgica no tratamento de IU. Em estudo realizado por Dedicação et al. (2009), no qual foi realizado um levantamento de 76 prontuários de mulheres com IU, verificou-se que $74,19 \%$ das mulheres acometidas por IUE e 50\% acometidas por IUM, já haviam sido submetidas a algum tipo de intervenção cirúrgica para o tratamento da patologia.

Feldner Junior et al. (2002) observaram que das 114 pacientes que estudou, $25(21,9 \%)$ haviam se submetido a cirurgias prévias para IU. Também Martins et al. (2000), ao analisarem em seu estudo os resultados cirúrgicos do tratamento com sling em 21 mulheres incontinentes, identificaram que 12 apresentavam cirurgia prévia para a patologia.

De acordo com Carramão et al. (2009), a conduta convencional no tratamento do prolapso uterino é a histerectomia vaginal, com finalidade de diminuir os riscos de recidivas. Entretanto, a histerectomia pode estar associada ao desenvolvimento de IU, em decorrência dos danos à inervação pélvica e às estruturas de suporte pélvico que podem ocorrer durantes a cirurgia (GUARISI et al., 2001b). Como o útero suporta parte do assoalho pélvico, sua remoção pode comprometer estruturas que sustentam a bexiga e a uretra. (HIGA, LOPES; REIS, 2008).

As diversas técnicas cirúrgicas têm altos índices de insucesso, e após alguns anos, o problema pode retornar. Ramos et al. (2000), citam em seu artigo que $100 \%$ das pacientes com cirurgias prévias para correção para IUE, tiveram recidiva nas três técnicas cirúrgicas (Burch, Marshall-MarchettiKrantz e Kelly-Kennedy) realizadas. Esse mesmo autor ainda conclui que, se deve melhor avaliar as pacientes do ponto de vista diagnóstico e repensar a indicação cirúrgica nos casos de recidiva.

Por muitos anos, a cirurgia foi considerada a melhor opção de tratamento da IU. Porém, as recidivas freqüentes acabavam submetendo as pacientes às mesmas condições iniciais, muitas vezes com piora do prognóstico, por isso as técnicas de tratamento conservador foram assumindo um papel importante no tratamento dessas pacientes. Contudo, no Brasil, a abordagem ainda é tradicionalmente cirúrgica (BELTRAMI et al., 2009; RETTI et al., 2007).

Quanto à abordagem fisioterapêutica durante o 
período de internamento hospitalar, verificou-se nos prontuários que $211(97,24 \%)$ pacientes não tiveram tipo algum de atendimento fisioterapêutico e em seis $(2,76 \%)$ havia relato da atuação da fisioterapia (Figura 4).

Figura 4 - Distribuição das pacientes de acordo com a abordagem fisioterapêutica durante a internação hospitalar. Cascavel, PR.

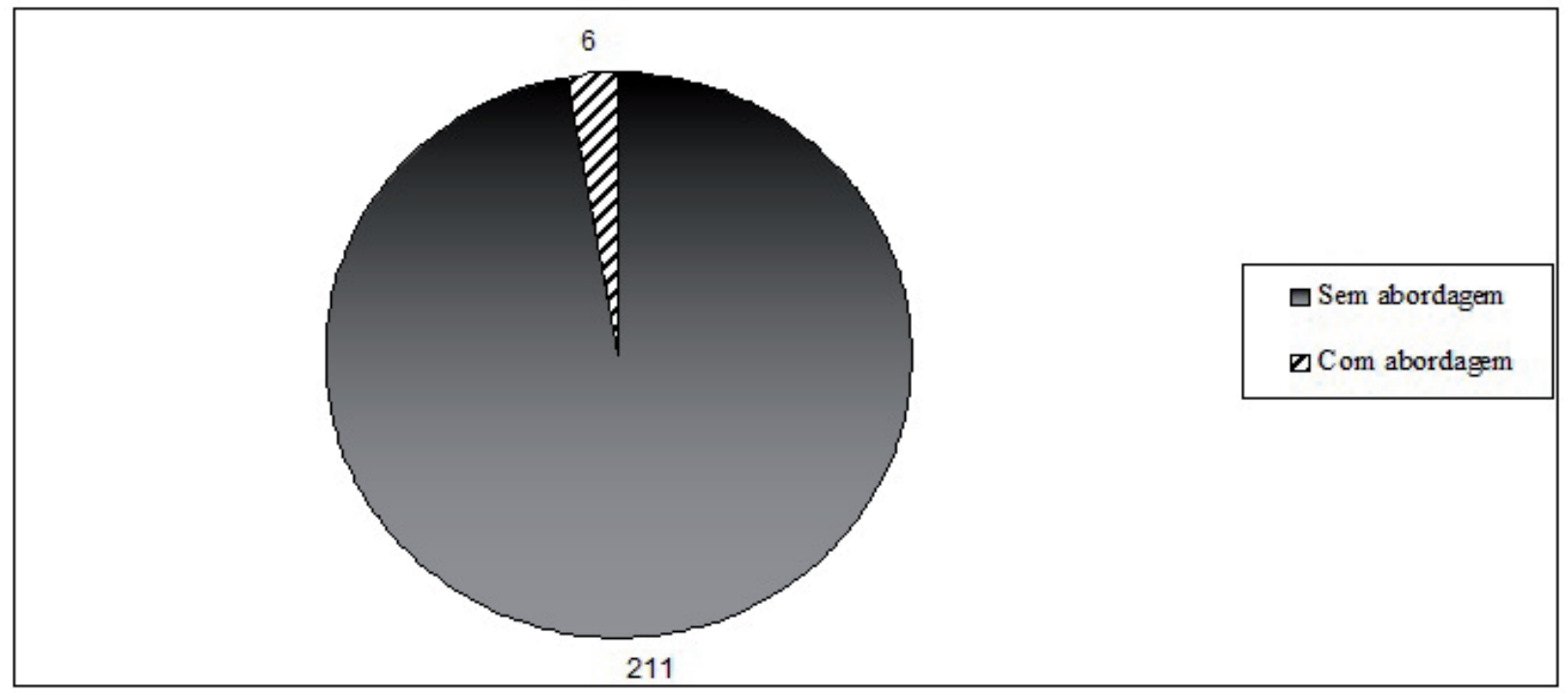

Vale ressaltar que, segundo os dados deste estudo, do total de pacientes submetidas à cirurgia, 85 $(39,17 \%)$ apresentavam prolapsos genitais de grau I, ou seja, em estados iniciais, sendo o tratamento conservador o mais indicado para sua correção, em especial em pacientes que ainda desejam ter filhos ou tenham algum tipo de contra-indicação cirúrgica (HAGEN, 2006).

Os resultados apresentados neste estudo mostram que não é comum o atendimento fisioterapêutico, preventivo e/ou pós-cirúrgico, às pacientes com IU submetidas a tratamento cirúrgico no HUOP. É importante ressaltar que, nos prontuários em que a atuação da fisioterapia foi mencionada, não foi especificado o tipo de abordagem terapêutica dado às pacientes.

Estudos mostram a eficácia das diferentes técnicas de tratamento fisioterapêutico em mulheres com IU. Bernardes et al. (2000) avaliaram 14 pacientes com IUE (sendo sete tratadas com cinesioterapia e sete submetidas à eletroestimulação endovaginal (EEEV) e verificaram que, após o tratamento com cinesioterapia, $71,4 \%$ pacientes estavam sem sintomas e $28,6 \%$ com perda leve. Já no grupo que foi submetido à EEEV, 28,6\% dos pacientes não apresentavam sintomas de IU, 57,1\% tinham perda leve e $14,3 \%$ perda moderada.

Rodrigues et al. (2005) utilizaram cinesioterapia e eletroestimulação do períneo associados à correção postural para o tratamento de três pacientes incontinentes e observaram que houve diminuição da perda de urina entre todas as participantes do estudo: a paciente 1 obteve controle urinário de $93,6 \%$, paciente 2 de $90,8 \%$ e a paciente 3 de $97,3 \%$.

Segundo Wall e Norton (1993), as chances de sucesso no tratamento da IU são muito maiores quando a paciente entende o procedimento e suas possíveis complicações e tem a oportunidade de 
considerar outras formas de terapia.

A fisioterapia no tratamento da IU vem mostrando resultados muito significativos, apresentando resolução e/ou melhora do problema em grande parte dos casos estudados, além de oferecer menos riscos às pacientes. No entanto, em nossa realidade, os serviços básicos de saúde não dispõem de programas de reabilitação do assoalho pélvico, que poderiam ser implantados e conduzidos por profissionais devidamente capacitados. Com isso, a população teria maior acesso a outras formas de tratamento, o que poderia evitar muitas técnicas cirúrgicas, minimizando os custos anuais com este tipo de procedimento e os casos de recidivas.

\section{Considerações Finais}

Por meio deste estudo, pode-se observar o predomínio de mulheres com IU atendidas no HUOP da cor branca, do lar, com idade entre 40 e 59 anos. O diagnóstico que prevaleceu entre as pacientes foi o de IUE, sendo a técnica de sling associada à CPP anterior o procedimento realizado na maioria. Somente em seis $(2,76 \%)$ prontuários havia relato da atuação da fisioterapia.

Por meio da análise dos resultados, pode-se concluir que, embora a fisioterapia tenha resultados significativos no tratamento da IU, ela ainda é pouco utilizada, mesmo nos lugares que dispõem deste tipo de atendimento. Sugere-se a realização de mais estudos a respeito do tema abordado, com o intuito de divulgar os riscos e benefícios dos diversos tratamentos para IU disponíveis.

\section{Referências}

ABRAMS, P. H.; CARDOZO, L.; KHOURY, S.; WEIN, A. Urinary incontinence: adult conservative management. In: INTERNATIONAL CONSULTATION COMMITTEE OF THE INTERNATIONAL CONTINENCE SOCIETY, 3., 2005, Montreal, Canadá. Anais... Montreal, 2005.

ABRAMS, P.; CARDOSO, L.; FALL, M.; GRIFFTHS, D.; ROSIER, P.; ULMSTEN, U. The standardization of terminology of lower urinary tract function: repot from the standardization sub-committee of the international continence society. Urology, Secaucus, v. 61, n. 1, p. $37-$ 49, 2003.

BELTRAMI, A. O.; STORTI, L. R.; MULLER, A.; CAMARGO, A. C. M.; GUARISI, T.; GOLLOP, T. R.; BORGES, J. B. R. Percepção das pacientes do ambulatório de uroginecologia quanto ao acolhimento e à abordagem terapêutica conservadora na incontinência urinária. Einstein, São Paulo, v. 7, n. 3, p. 328-333, set. 2009.

BERNARDES, N. O.; PÉRES, F. R.; SOUZA, E. L. B. L.; SOUZA, O. L. Métodos de tratamento utilizados na incontinência urinária de esforço genuína: um estudo comparativo entre cinesioterapia e eletroestimulação endovaginal. Revista Brasileira de Ginecologia e Obstetrícia, Rio de Janeiro, v. 22, n. 1, p. 49-54, jan./ fev. 2000.

BROWN, J. S.; GRADY, D.; OUSLANDER, J. G.; HERZOG, A. R.; VARNER, R. E.; POSNER, S. F. Prevalence of urinary incontinence and associated risk factors in postmenopausal women. Obstetrics and Gynecology, New York, v. 94, n. 1, p. 66-70, jul. 1999.

CARRAMÃO, S.; AUGE, A. P. F.; PACETTA, A. M.; DUARTE, E.; AYROSA, P.; LEMOS, N. L. M. L.; AOKI, T. Estudo randômico da correção cirúrgica do prolapso uterino através da tela sintética de polipropileno tipo I comparando histerectomia versus preservação uterina. Revista do Colégio Brasileiro de Cirurgiões, Rio de Janeiro, v. 36, n. 1, p. 65-72, jan./fev. 2009.

DEDICAÇÃO, A. C.; HADDAD, M.; SALDANHA, M. E. S.; DRIUSSO, P. Comparação da qualidade de vida nos diferentes tipos de incontinência urinária. Revista Brasileira de Fisioterapia, São Carlos, v. 13, n. 2, p. 116122, mar./abr. 2009.

FELDNER JUNIOR, P. C.; BEZERRA, L. R. P. S.; GIRÃO, M. J. B. C.; CASTRO, R. A. C.; SARTORI, M. G. F.; BARACAT, E. C.; LIMA, G. R. Valor da queixa clínica e exame físico no diagnóstico da incontinência urinária. Revista Brasileira de Ginecologia e Obstetrícia, Rio de Janeiro, v. 24, n. 2, p. 87-91, mar. 2002.

FIGUEIREDO, E. M.; LARA, J. O.; CRUZ, M. C.; QUinTÃO, D. M. G.; MONTEIRO, M. V. C. Perfil sociodemográfico e clínico de usuárias de serviço de fisioterapia uroginecológica da rede pública. Revista Brasileira de Fisioterapia, São Carlos, v. 12, n. 2, p. 136142, mar./abr. 2008.

GONÇALVES, J. G.; OLIVEIRA, L. M.; FREITAS, M. M. S.; BARACAT, E. C. Prolapso genital. In: GIRÃO, M. J. B. C.; RODRIGUES DE LIMA, G.; BARACAT, 
E. C. (Ed.). Uroginecologia. São Paulo: Artes Médicas, 1997. p. 143-149.

GRODSTEIN, F.; FRETTS, R.; LIFFORD, K.; RESNICK, N.; CURHAN, G. Association of age, race, and obstetric history with urinary symptoms among women in the nurses' health study. American Journal of Obstetrics and Gynecology, Saint Louis, v. 189, n. 2, p. 428-434, ago. 2003.

GUARISI, T.; PINTO-NETO, A. M.; OSIS, M. J.; PEDRO, A. O.; COSTA-PAIVA, L. H. S.; FAÚDES, A. Procura de serviço médico por mulheres com incontinência urinária. Revista Brasileira de Ginecologia e Obstetrícia, Rio de Janeiro, v. 23, n. 7, p. 439-443, ago. 2001a.

GUARISI, T.; PINTO-NETO, A. M.; OSIS, M. J.; PEDRO, A. O.; COSTA-PAIVA, L. H. S.; FAÚDES, A. Incontinência urinária entre mulheres climatéricas brasileiras: inquérito domiciliar. Revista de Saúde Pública, São Paulo, v. 35, n. 5, p. 428-435, out. 2001b.

HAAB, F.; LEACH, G. E. Suporte pubo-vaginal: procedimentos de colocação. In: RUBINSTEIN, I. Urologia Feminina. São Paulo: BYK, 1999. p. 256-261.

HAGEN, S.; STARK, D.; MAHER, C.; ADAMS, E. Conservative management of pelvic organ prolapse in women. The Cochrane Database of Systematic Reviews, England, v. 18, n. 4, p. 45-51, out. 2006.

HIGA, R.; LOPES, M. H. B. M.; REIS, M. J. Fatores de risco para a incontinência urinária na mulher. Revista da Escola de Enfermagem da USP, São Paulo, v. 42, n. 1, p. 187-192, mar. 2008.

LOPES, M. H.; HIGA, R. Restrições causadas pela incontinência urinária à vida da mulher. Revista da Escola de Enfermagem da USP, São Paulo, v. 40, n. 1, p. 34-41, mar. 2006.

MARTINS, J. A. M.; CASTRO, R. A.; GIRÃO, M. J. B. C.; SARTORI, M. G. F.; BARACAT, E. C.; LIMA, G. R. Correção da incontinência urinária de esforço com sling: resultados iniciais. Revista Brasileira de Ginecologia e Obstetrícia, Rio de Janeiro, v. 22, n. 5, p. 301-305, jun. 2000.

MATHEUS, L. M.; MAZZARI, C. F.; MESQUITA, R. A.; OLIVEIRA, J. Influência dos exercícios perineais e dos cones vaginais, associados à correção postural, no tratamento da incontinência urinária feminina. Revista Brasileira de Fisioterapia, São Carlos, v. 10, n. 4, p. 387 392, out./dez. 2006.

MELONI, W. A. B.; TOMÉ, A. L. F.; JUNIOR, A. J. K.; OLIVEIRA, A. C.; BESTANE, M. C.; BESTANE, W. J. Tratamento cirúrgico da incontinência urinária de esforço feminina com sling pubovaginal: há melhora da qualidade de vida? Revista Médica Ana Costa, São Paulo, v. 9, n. 1, jan./mar. 2004.

MOLLER, L. A.; LOSE, G.; JORGENSEN, T. Risk factors for lower urinary tract symptoms in women 40 to 60 years of age. Obstetrics and Gynecology, New York, v. 96 , n. 3, p. 446-451, set. 2000.

OLIVEIRA, W. L.; GUN, L. G.; GONZALES, F. L. M.; GUN. S. Incontinência urinária tratamento pela técnica do sling pubovaginal: revisão de 120 casos. Revista Farmacêutica de Ciências Médicas, Sorocaba, v. 5, n. 2, p. 25-27, 2003.

RAMOS, J. G. L.; XAVIER, N. L.; NÁCUL, A. P.; ZUCATTO, A. E.; HENTSCHEL, E. L. Comparação dos resultados do tratamento cirúrgico da incontinência urinária de esforço por três diferentes técnicas cirúrgicas. Revista Brasileira de Ginecologia e Obstetrícia, Rio de Janeiro, v. 22, n. 1, p. 43-48, jan./fev. 2000.

RETTI, M. T.; SIMÕES, J. A.; HERRMANN, V.; GURGELIII, M. S. C.; MORAIS, S. S. Qualidade de vida em mulheres após tratamento da incontinência urinária de esforço com fisioterapia. Revista Brasileira de Ginecologia e Obstetrícia, Rio de Janeiro, v. 29, n. 3, p. 134-140, mar. 2007.

RODRIGUES, N. C.; SCHERMA, D.; MESQUITA, R. A.; OLIVEIRA, J. Exercícios perineais, eletroestimulação e correção postural na incontinência urinária - estudo de casos. Fisioterapia em Movimento, Curitiba, v. 18, n. 3, p. 23-29, jul./set. 2005.

SARTORI, J. P.; MARTINS, J. A. M.; CASTRO, R. A.; SARTORI, M. G. F.; GIRÃO, M. J. B. C. Sling de aponeurose e com faixa sintética sem tensão para o tratamento cirúrgico da incontinência urinária de esforço feminina. Revista Brasileira de Ginecologia e Obstetrícia, Rio de Janeiro, v. 30, n. 3, p. 127-134, 2008.

SILVA, L.; LOPES, M. H. B. M. Incontinência urinária em mulheres: razões para a não procura por tratamento. Revista da Escola de Enfermagem da USP, São Paulo, v. 43, n. 1, p. 72-78, mar. 2009.

SILVEIRA, A. B. F. N.; DOUGLAS FOGIATTO, D.; KULAK JÚNIOR, J. K.; BUSATO, D.; FRANCISCO, J. A. F. C. Sling transobturatório: resultados de um centro de uroginecologia em Curitiba no ano de 2004. Revista do Colégio Brasileiro de Cirurgiões, Rio de Janeiro, v. 34, n. 2, p.123-6, mar./ abr. 2007.

SOUZA, C. E. C.; LIMA, R. M.; BEZERRA, L. M. A.; PEREIRA, R. W.; MOURA, T. K.; OLIVEIRA, R. J. Estudo comparativo da função do assoalho pélvico em mulheres continente e incontinentes na pós menopausa. Revista Brasileira de Fisioterapia, São Carlos, v. 13, n. 
6, p. 535-541, nov. 2009.

TEIXEIRA, A.; GUIMARÃES, C. A.; ZIMER, S. R. A.; OLIVEIRA, A. P. M. V. Tratamento fisioterapêutico na incontinência urinária de esforço em mulheres de 35 a 55 anos. Revista Unicenp de Biologia e Saúde, Curitiba, v. 1, n. 3, p. 12-16, abr./jun. 2005.

WALL, L. L. Incontinência, prolapso e distúrbios do assoalho pélvico. In: BEREK, J.; ADASHI, E. Y.; HILLARD, E. A. Tratado de Ginecologia. 12. ed. Rio de Janeiro: Guanabara Koogan, 1998. p. 437.

WALL, L. L.; NORTON, P. A. Surgical management of stress incontinence. In: . (Ed.). Practical urogynecology. Baltimore: Williams \& Wilkins, 1993. p. 153-190.

ZUCHI, E. V. M.; KATI, L. M.; GIRÃO, M. J. B. C.; SARTORI, M. G. F.; BARACAT, E. C.; LIMA, G. R. Colpossacrofixação para correção do prolapso da cúpula vaginal. Revista Brasileira de Ginecologia e Obstetrícia, Rio de Janeiro, v. 25, n. 10, p. 705-709, nov./dez. 2003.

Recebido em 14 de março de 2011 - Received on March 14, 2011

Aceito em 12 de julho de 2011 - Accepted on July 12, 2011 
Frare, J. C.; Souza, F. T.; Silva, J. R. 